\title{
QTL for body weight, morphometric traits and stress response in European sea bass Dicentrarchus labrax
}

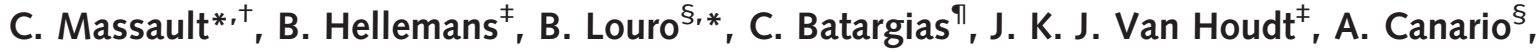 \\ F. A. M. Volckaert ${ }^{\ddagger}$, H. Bovenhuis ${ }^{\dagger}$, C. Haley* and D. J. de Koning* \\ *Division of Genetics and Genomics, Roslin Institute and Royal (Dick) School of Veterinary Sciences, University of Edinburgh, Roslin, \\ Midlothian EH25 9PS, UK. ${ }^{\dagger}$ Animal Breeding and Genetics Group, Wageningen University, PO Box 338, Wageningen, NL-6700AH, \\ The Netherlands. ${ }^{\ddagger}$ Laboratory of Animal Diversity and Systematics, Katholieke Universiteit Leuven, Ch. Deberiotstraat 32, B-3000 Leuven, \\ Belgium. ${ }^{5}$ Centre of Marine Sciences (CCMAR), University of Algarve, Gambelas, 8005-139 Faro, Portugal." Laboratory of Applied Fish \\ Genetics, Department of Aquaculture \& Fisheries Management, Technological Educational Institute of Messolonghi, Nea Ktiria, 30200 \\ Messolonghi, Greece
}

\section{Summary}

\begin{abstract}
Natural mating and mass spawning in the European sea bass (Dicentrarchus labrax L., Moronidae, Teleostei) complicate genetic studies and the implementation of selective breeding schemes. We utilized a two-step experimental design for detecting QTL in mass-spawning species: 2122 offspring from natural mating between 57 parents ( 22 males, 34 females and one missing) phenotyped for body weight, eight morphometric traits and cortisol levels, had been previously assigned to parents based on genotypes of 31 DNA microsatellite markers. Five large full-sib families (five sires and two dams) were selected from the offspring (570 animals), which were genotyped with 67 additional markers. A new genetic map was compiled, specific to our population, but based on the previously published map. QTL mapping was performed with two methods: half-sib regression analysis (paternal and maternal) and variance component analysis accounting for all family relationships. Two significant QTL were found for body weight on linkage group 4 and 6, six significant QTL for morphometric traits on linkage groups 1B, 4, 6, 7, 15 and 23 and three suggestive QTL for stress response on linkage groups 3, 14 and 23. The QTL explained between $8 \%$ and 38\% of phenotypic variance. The results are the first step towards identifying genes involved in economically important traits like body weight and stress response in European sea bass.
\end{abstract}

Keywords aquaculture, body weight, cortisol, Dicentrarchus labrax, European sea bass, morphometry, QTL mapping, selection, stress response.

\section{Introduction}

European sea bass (Dicentrarchus labrax L., Moronidae, Teleostei) is a marine fish, commonly distributed along the warm temperate coasts of the south-eastern Atlantic Ocean and Mediterranean Sea. While in 1980 only 10 tonnes were produced in semi-intensive aquaculture systems, production has stabilized since 2000 and the total worldwide production was an estimated 105900 tonnes in 2008 (http://

\section{Address for correspondence}

C. Massault, Division of Genetics and Genomics, Roslin Institute and Royal (Dick) School of Veterinary Sciences, University of Edinburgh, Roslin, Midlothian EH25 9PS, UK.

E-mail: Cecile.massault@wur.nl

Accepted for publication 8 November 2009 www.globefish.org). The value of sea bass aquaculture totalled 320 million Euros in 2007, at an average value of $\$ 5.1$ per $\mathrm{kg}$. Greece is the main producer $(44 \%)$, followed by Turkey (26\%), Italy (12\%) and Spain (8\%), and sea bass represent $40 \%$ of Mediterranean aquaculture (http:// www.feap.info).

Although sea bass aquaculture started 30 years ago, selective breeding is not widely used (Vandeputte et al. 2001). For a long time, natural mating and mass spawning were commonly used for reproduction. Artificial reproduction and mating are now fully controlled (Moretti 1999; Saillant et al. 2001; Dupont-Nivet et al. 2006) and have gradually become standard operational practice. In the case of mass selection, the highly skewed unequal contribution of parents that is so typical of mass-spawning fish (Jones \& Hutchings 2002; Herlin et al. 2008) leads to inbreeding. Furthermore, the unknown pedigree of the individuals 
complicates selective breeding. However, the use of information from QTL is a first step towards selective breeding. Regions of the genome that are linked to a quantitative trait of interest are detected using QTL mapping.

The steady increase in genetic and molecular biological studies of European sea bass (for a review, see Volckaert et al. 2008) provides a platform to implement breeding programmes that do not depend solely on the measurement of phenotypic traits, but rely also on information from genetic markers, i.e. marker assisted selection. Large scale profiling of paternity with microsatellite markers has facilitated the breeding of families on an experimental and commercial scale in a reliable and affordable fashion (García de Léon et al. 1995; Chatziplis et al. 2007). From such communally bred families, quantitative genetic parameters have been reported for heritabilities and phenotypic and genotypic correlations for some traits, such as sex (Vandeputte et al. 2007), body weight and length (Vandeputte \& Launey 2004; Saillant et al. 2006; Chatziplis et al. 2007) and carcass traits (Saillant et al. 2009). Heritabilities for growth are relatively high and vary from 0.29 to 0.60 in European sea bass (Saillant et al. 2006; Dupont-Nivet et al. 2008). These high heritabilities have allowed a doubling in growth rate in just four generations (B. Chatain, personal communication). A medium density genetic map is available and has been updated (Chistiakov et al. 2005, 2008), based on more than 200 microsatellites and more than 200 amplified fragment length polymorphisms (AFLPs). The ESTs of several tissue-specific cDNA banks have been sequenced, analysed (A. Canario, pers. comm.), and used as a source of microsatellite (Chistiakov et al. 2008) and SNP markers (Souche 2009). At the same time, a QTL analysis for body weight has been performed in European sea bass (Chatziplis et al. 2007) and has identified a QTL for growth on linkage group 1.

An important economical trait is stress, either linked to pathogen infection or behaviour (such as confinement and handling). Cortisol, a widely accepted proxy for stress in fish, affects the immune system response (Engelsma et al. 2002). Therefore, if genomic regions are found that influence the stress response, this could have a beneficial effect on the management of fitness. All these features represent multiple steps towards the use of genetic and molecular tools for improvement of production.

Massault et al. (2008) suggested a two-step experimental design for QTL mapping in mass-spawning species with natural mating. In the first step, individuals are assigned to parents using a limited number of markers, and in a second step a subset of large families is selected for a whole genome scan. So far, such a design has not been applied in practice to fish. The strategy has been implemented in a single study on European sea bass. Volckaert et al. (submitted) described how the families for QTL analysis were established by assigning parentage among the progeny of a 1-day batch spawning. Heritabilities for growth, morphometry and stress response were calculated on these data and are reported by Volckaert et al. (submitted). The aim of this study was to perform the QTL analysis on stress response, body weight and morphological traits.

\section{Materials and methods}

\section{Experiment}

We followed a two-step procedure for the stress experiment that takes into account the specificity of the natural mating population, as suggested by Massault et al. (2008): (i) take a random sample of 2122 offspring, originating from a single day natural mating by mass spawning and assign parents using a limited number of genetic markers, (ii) select large families for further genotyping and QTL mapping.

Fifty-seven parents originating from commercial and wild lines made up the base population. Offspring were chosen at random and genotyped for 31 loci at 50 days of age. Based on these genotypes, offspring were assigned to parents. Confinement stress was induced on all 2122 fish at approximately 8 months old, by reducing the volume of water to $0.2 \mathrm{~m}^{3} / \mathrm{kg}$ of fish for $4 \mathrm{~h}$ (for details see Volckaert et al. submitted).

\section{Phenotyping}

Following the stress experiment, individuals were phenotyped for ten traits: body weight, eight various morphometric traits, and cortisol level (for full details see Volckaert et al. submitted; see Fig. 1 and Table 1). About half of the offspring were stunned in the morning using icy water whereas the other half were stunned in the afternoon. After being stunned, each fish was bled, weighted and digitally photographed. The plasma, after separation from the red blood cells, was conserved at $-20{ }^{\circ} \mathrm{C}$. When photographed, fish were placed as laterally as possible to avoid shape variation, and landmarks were placed at various points on the body after determining the common scale using a ruler

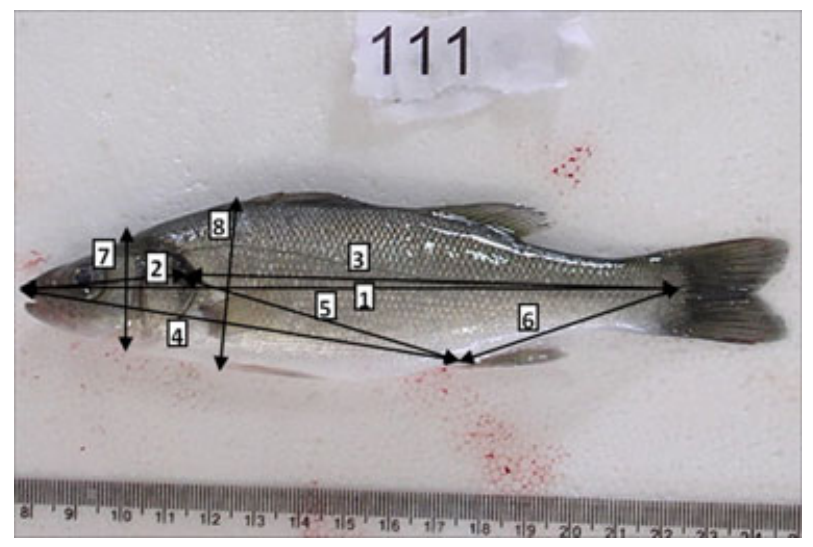

Figure 1 Morphometric measurement of European sea bass. Numbers are related to the traits described in Table 1. 
Table 1 Number of observations, mean, standard deviation, coefficient of variation and heritabilities (standard error) for 10 traits in 570 European sea bass.

\begin{tabular}{|c|c|c|c|c|c|c|}
\hline Trait & Abbreviation* & No. individuals & Mean & SD & $\mathrm{CV}(\%)$ & $\mathrm{h}^{2 * *}( \pm \mathrm{SE})$ \\
\hline Body weight & BW (-) & 566 & 40.58 & 14.21 & 35 & $0.54( \pm 0.2)$ \\
\hline Cortisol & CORT (-) & 444 & 319.5 & 136.40 & 43 & $0.08( \pm 0.06)$ \\
\hline 1. Standard length & $\mathrm{SL}(\mathrm{SL})$ & 540 & 13.26 & 1.61 & 12 & $0.65( \pm 0.22)$ \\
\hline 2. Head length & HL (SNOP) & 540 & 3.77 & 0.46 & 12 & $0.61( \pm 0.21)$ \\
\hline 3. Body length & $\mathrm{BL}(\mathrm{OPCA})$ & 540 & 9.50 & 1.17 & 12 & $0.64( \pm 0.22)$ \\
\hline 4. Pre anal length & PrAnl (SNAN) & 540 & 9.20 & 1.16 & 13 & $0.68( \pm 0.23)$ \\
\hline 5. Abdominal length & AL (OPAN) & 540 & 5.57 & 0.72 & 13 & $0.66( \pm 0.23)$ \\
\hline 6. Post-anal length & PsAnl (ANCA) & 540 & 4.46 & 0.54 & 12 & $0.52( \pm 0.20)$ \\
\hline 7. Head depth & $\mathrm{HD}(-)$ & 540 & 2.46 & 0.33 & 13 & $0.64( \pm 0.22)$ \\
\hline 8. Body depth & $\mathrm{BD}$ (DOPV) & 540 & 3.38 & 0.41 & 12 & $0.56( \pm 0.21)$ \\
\hline
\end{tabular}

${ }^{*}$ Abbreviations according to Chatziplis et al. 2007.

**Heritabilities calculated from 922 fishes (including QTL population) in Volckaert et al. (submitted).

and coordinated axes (x,y and $\mathrm{z}$ ). From those landmarks, morphometric traits were observed.

The cortisol level was determined using radio immunoassay technology (RIA) based on antiserum raised in rabbit against cortisol-3-CMO-BSA and tritriated cortisol (Fitzgerald Industries International). RIA cross-reacted with only one plasma component, co-migrating with cortisol in layer chromatography. Plasma samples were diluted in phosphate buffer containing $0.5 \mathrm{~g} / \mathrm{l}$ gelatine $(\mathrm{pH} 7.6)$ and heated at $80{ }^{\circ} \mathrm{C}$. We extracted the cortisol according to the protocol of Scott et al. (1982), as detailed in Volckaert et al. (submitted).

\section{Genotyping and genetic map}

Volckaert et al. (submitted), using a triple multiplex-PCR of 31 microsatellite markers, identified 11 large full-sib families $(n=922)$ from the 2122 offspring for heritability and correlation analysis (see above). Five of the eleven families ( $n=570$ fish) and their seven parents were fully genotyped for 98 microsatellite markers (with eight markers not fulfilling minimal quality requirements). The population structure for the QTL mapping includes the offspring from five males and two females; the number of offspring per family varies between 93 and 143 (Table 2). We generated a genetic map specific to our population using the software CRI-MAP V.2.4. (http://compgen.rutgers.edu/multimap/ crimap).

\section{QTL mapping}

We used two methods to detect QTL: half-sib regression analysis as described by Knott et al. (1996), available on the web (http://www.gridqtl.org.uk, Hernandez \& Knott 2009), and variance component analysis (VCA) as described by George et al. (2000) (http://qtl.cap.ed.ac.uk/puccinoserv lets/hkloaderLoki). The model used for half-sib analyses is as follows:
Table 2 The structure of the QTL population of European sea bass. Five males were mated with two females, providing full-sib families and paternal and maternal half-sib families.

\begin{tabular}{lllll}
\hline Half-sib ID & Full-sib ID & Dam ID & Sire ID & No. offspring \\
\hline 1 & 1 & 2 & 1 & 98 \\
1 & 2 & 2 & 3 & 93 \\
2 & 3 & 6 & 4 & 92 \\
2 & 4 & 6 & 5 & 143 \\
2 & 5 & 6 & 7 & 142 \\
\hline
\end{tabular}

$$
y_{i j}=\alpha_{i}+\beta_{i} x_{i j}+e_{i j} \text {, }
$$

where $y_{i j}$ is the offspring phenotype, $\alpha_{i}$ the mean for family $i$, $\beta_{i}$ the regression coefficient for family $i, x_{i j}$ the probability of inheriting parental allele 1 conditional on the marker information and $e_{i j}$ the residual. Information content (IC) was calculated as described by Knott et al. (1996). The QTL effect is estimated within families as the allele substitution effect. Knott et al. (1996) provide a method to calculate the QTL effect across families in terms of variance because of the QTL by looking at the difference between the residuals of full and reduced models:

$$
\text { QTL effect }=4 \times(1-(\text { RES full/RES reduced })),
$$

where RES full and RES reduced are the residuals of full and reduced models respectively.

Regression was performed at each centiMorgan and the test statistic for presence of a QTL was calculated as described by Knott et al. (1996). We used permutation tests with 500 iterations and bootstrap analysis with 2000 iterations to evaluate the significance and confidence intervals of detected QTL respectively. A QTL is considered significant if it exceeds the 5\% genome-wide threshold and a QTL is considered suggestive if it exceeds the 5\% chromosome-wide threshold.

For VCA, we used the genotypes and the linkage map to first calculate the identity-by-descent (IBD) matrix at each 
position of the genome with Loki (Heath 1997). In the analysis, only linkage information on the transmission of alleles from parent to offspring was considered and IBD relationships between parental alleles were not considered (i.e. no linkage disequilibrium). The resulting IBD matrix is used to model a QTL in a linear mixed model using ASREML:

$$
y=X b+Z u+Z v+e,
$$

where $y$ is the phenotype, $X$ is the incidence matrix relating phenotypes to systematic environmental effects, $b$ is the vector with solutions for systematic environmental effects, $Z$ the incidence matrix relating animals to phenotype, $u$ vector of additive polygenic effect, $v$ vector of additive QTL effect and $e$ vector of environmental effect $\left(\operatorname{var}(u)=A \sigma^{2} u\right.$ and $(v)=G \sigma^{2} v$, with A the additive relationship matrix and $\mathrm{G}$ the IBD matrix). For CORT, the environmental effect of sampling time was included. The sampling time corresponds to a half-day, resulting in three classes: first day morning, first day afternoon and second day morning. For the other traits the only other systematic effect was the mean. The QTL effect is given by the VCA as the proportion of phenotypic variance because of the QTL.

The null hypothesis model is:

$$
y=X b+Z u+e
$$

with the same variable definitions as for the QTL model. The presence of a QTL, treated as a random effect, was tested at every centiMorgan using the likelihood ratio test:

$$
\mathrm{LRT}=2 \times(l f-l r),
$$

where LRT is the log likelihood ratio test and If is the $\log$ likelihood of the full model i.e. the model including the QTL and $I r$ the log likelihood from the reduced model i.e. the model without a QTL. Under the null hypothesis, the likelihood ratio test follows a chi-square distribution with one degree of freedom. For VCA, we computed 1\% chromosomewide thresholds according to the method described by Piepho (2001). The thresholds vary as a function of the chromosome length and the trait studied.

\section{Results}

\section{Summary statistics}

Heritabilities for each trait (listed in Table 1 and including abbreviations) were calculated for 922 animals, including the QTL population (Volckaert et al. submitted), while all other statistics were derived from the actual QTL population ( $n=570)$. CORT heritability is 0.08 , while heritabilities of the others traits ranged from 0.52 to 0.68 . We note that all morphometric traits (SL, HL, BL, PRAL, AL, POAL, HD and $\mathrm{BD})$ have a coefficient of variation $(\mathrm{CV})$ within the same range (12-13\%), while $\mathrm{BW}$ and CORT are more variable (CV of $35 \%$ and $43 \%$ respectively). Morphometric traits are highly positively correlated among themselves and with BW (see also Volckaert et al. submitted).

\section{Genetic map}

Based on the marker genotypes collected from 570 offspring and their parents, we built a genetic map containing 20 linkage groups and covering $639 \mathrm{cM}$ (Fig. 2). Of the 90 markers, 87 were located in linkage groups consisting of two or more markers. Three markers were unlinked to any of the other markers (linkage group LG9, LG18 and LG25). The average marker spacing is $7.70 \mathrm{cM}$. The LG1 was cut into two parts (1a and $1 \mathrm{~b}$ ) because of a gap $>100 \mathrm{cM}$. LG16, LG21 and LG22 are not represented in this genetic map as no markers were located on them.

\section{Information content}

Table 3 summarizes the average IC of each linkage group for both paternal and maternal half-sib (PHS and MHS) analysis, as well as their number of markers, average number of alleles and their length. The average IC of the genome amounts to 0.77 for PHS and 0.81 for MHS: it varied from 0.42 to 0.96 for PHS and 0.64 to 0.97 for MHS. We observed an average IC lower than 0.5 on LG2 and LG4 for both PHS and MHS. Another region on linkage group LG20 had low IC for PHS only.

\section{QTL detected}

Variance component analysis and regression analysis were performed for each trait for the whole genome (on the 20 linkage groups). Regression analysis was divided into PHS and MHS analysis (Table 4 and Fig. 3). Significant QTL for regression analysis were found when the F-statistics were above the 5\% genome-wide threshold and suggestive when above the $5 \%$ chromosome-wide thresholds, given by GridQTL and significant when log likelihood (LR) is above the $1 \%$ chromosome-wide threshold.

There were two BW QTL on LG4 and on LG6. The one on LG4 was present and highly significant in all analyses, although it was placed at different positions on the chromosome ( $\mathrm{F}=5.65$ for PHS with significance thresholds of $5 \% \mathrm{CW}$ of 3.10 and 5\% GW of 4.48, F = 7.09 for MHS with significance thresholds of $5 \% \mathrm{CW}$ of 3.76 and $5 \% \mathrm{GW}$ of 7.02 and $\mathrm{LR}=5.78$ for VCA with a significance threshold of $1 \% \mathrm{CW}$ of 13.5$)$. It was not possible to estimate the variation because of the BW QTL on LG4 detected with VCA, as the likelihood converged at the boundary of the parameter space, i.e. at a heritability of that QTL of 0.99, but the other analyses indicated a large QTL effect on this trait. Another large BW QTL was detected on LG6 with the MHS method ( $\mathrm{F}=8.59$ with $5 \% \mathrm{CW}$ of 3.82 and $5 \% \mathrm{GW}$ of 7.02 ).

There was a large number of highly significant morphology QTL, in particular for the PHS analysis. A common 


\section{LG1a}

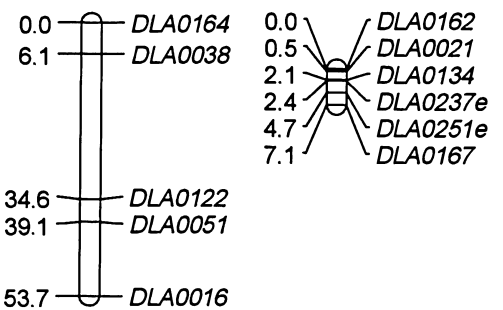

LG2

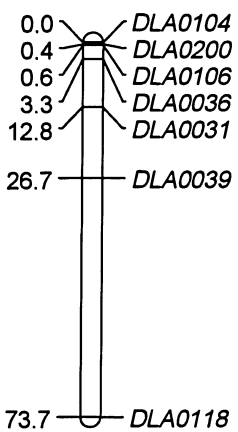

LG3

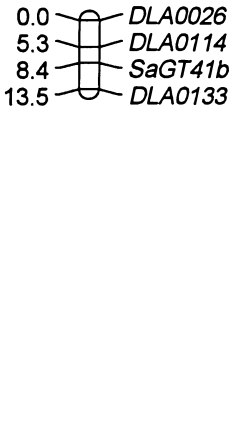

LG4

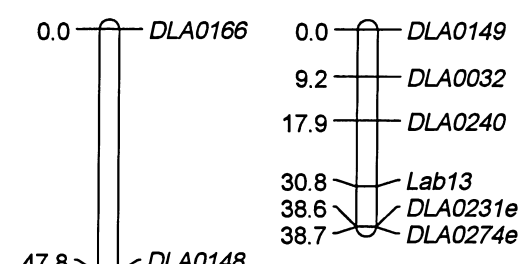

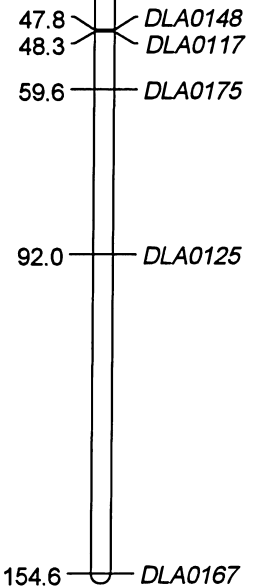

$1546-$ DLA0167

\section{LG6}

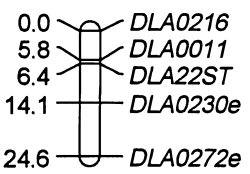

LG13

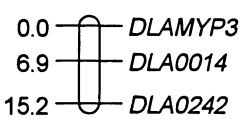

LG7

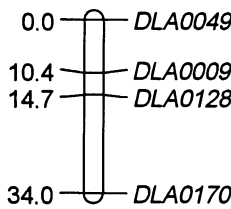

LG8

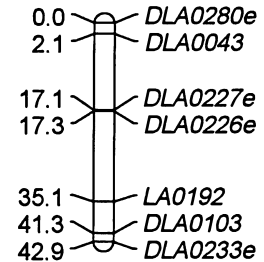

LG10

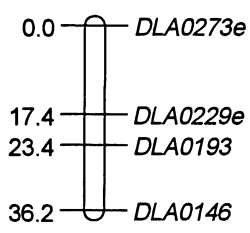

LG11 0.0 DLAO267e
$5.1-$ DLAO041

\section{LG12}

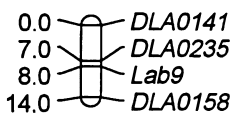

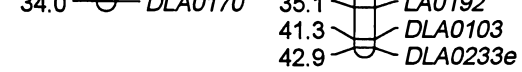

LG14

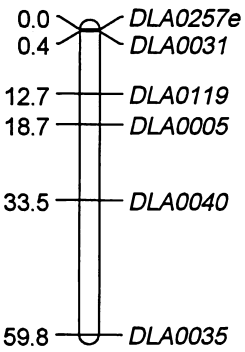

LG15

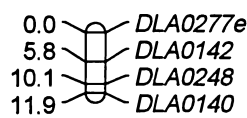

LG17



LG19

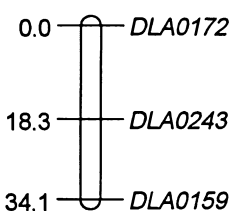

\section{LG20}

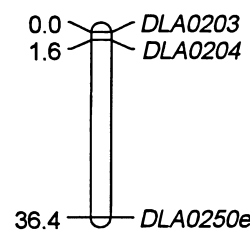

\section{LG23 LG24}

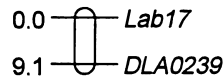

$0.0 \multimap$ DLAO029
$6.4 \bigcirc$ DLAO008

Figure 2 Genetic map of QTL-mapping sea bass population. The linkage groups LG16, LG21 and LG22 from Chistiakov et al. (2005) are missing as no markers from those groups are present.

QTL was found in LG4 by all analyses, but again at different positions ( $\mathrm{F}=4.76$ for PHS with $5 \% \mathrm{CW}$ of 2.90 and $5 \%$ GW of $4.18, \mathrm{~F}=10.31$ with $5 \% \mathrm{CW}$ of 4.37 and $5 \% \mathrm{GW}$ of 6.98 and LR $=15.46$ for VCA with $1 \% C W$ of 13.03). VCA showed a large proportion of phenotypic variation explained by the QTL (38\%), while this proportion was less for PHS and MHS (13\% and 14\% respectively). Another morphology QTL was detected with VCA on LG6 with a QTL effect of about $9.4 \%(\mathrm{LR}=22.79$ with $1 \% \mathrm{CW}$ of 12.07$)$. The other morphology QTL found were located on four different chromosomes and discovered with PHS analysis (F between 4.36 and 4.96 with $5 \% \mathrm{CW}$ of $2.41-2.69$ and $5 \%$ GW of 4.18). The QTL effects explained between $12 \%$ and $16 \%$ of phenotypic variation.

Three QTL for CORT were detected with PHS (LG3 and LG14, F $=2.69$ with $5 \% \mathrm{CW}$ of 2.70 and $\mathrm{F}=3.01$ with $5 \%$ 
Table 3 Number of markers, average number of alleles per marker, length of the linkage group, and average information content for paternal and maternal half-sib regression analysis (PHS and MHS) of European sea bass.

\begin{tabular}{lllrll}
\hline & No. & $\begin{array}{l}\text { Average no. } \\
\text { llleles }\end{array}$ & Length & $\begin{array}{l}\text { Avg IC } \\
\text { PHS }\end{array}$ & $\begin{array}{l}\text { Avg IC } \\
\text { MHS }\end{array}$ \\
\hline LG1a & 5 & 5.2 & 53 & 0.80 & 0.76 \\
LG1b & 6 & 4.3 & 7 & 0.96 & 0.97 \\
LG2 & 7 & 3.9 & 73 & $0.70^{1}$ & $0.68^{4}$ \\
LG3 & 4 & 3.5 & 13 & 0.83 & 0.81 \\
LG4 & 6 & 3.7 & 106 & $0.59^{2}$ & $0.64^{5}$ \\
LG5 & 6 & 4 & 45 & 0.88 & 0.91 \\
LG6 & 5 & 3.8 & 24 & 0.81 & 0.80 \\
LG7 & 4 & 6 & 34 & 0.89 & 0.84 \\
LG8 & 7 & 4.3 & 42 & 0.85 & 0.88 \\
LG10 & 4 & 4.3 & 36 & 0.83 & 0.80 \\
LG11 & 2 & 3.5 & 5 & 0.86 & 0.67 \\
LG12 & 4 & 5.5 & 14 & 0.92 & 0.90 \\
LG13 & 3 & 5 & 15 & 0.92 & 0.87 \\
LG14 & 6 & 3.8 & 59 & 0.79 & 0.77 \\
LG15 & 4 & 3 & 11 & 0.82 & 0.81 \\
LG17 & 3 & 4.8 & 17 & 0.89 & 0.92 \\
LG19 & 3 & 4 & 34 & 0.82 & 0.67 \\
LG20 & 3 & 3 & 36 & $0.42^{3}$ & 0.62 \\
LG23 & 2 & 4.5 & 9 & 0.88 & 0.92 \\
LG24 & 2 & 7 & 0.93 & 0.97 \\
\hline & & & & & \\
\hline
\end{tabular}

${ }^{1}$ Average of 0.49 between 50 and $46 \mathrm{cM}$.

${ }^{2}$ Average of 0.27 between 0 and $34 \mathrm{cM}$.

${ }^{3}$ Average of 0.32 between 12 and $36 \mathrm{cM}$.

${ }^{4}$ Average of 0.46 between 44 and $61 \mathrm{cM}$.

${ }^{5}$ Average of 0.37 between 0 and $33 \mathrm{cM}$.

CW of 2.74 respectively) and MHS (LG23, F $=6.59$ with $5 \% \mathrm{CW}$ of 3.25 ) and were $5 \%$ significant chromosome-wide. These were therefore considered as suggestive QTL. None were detected with VCA. The proportion of phenotypic variation explained by the QTL varied between $8 \%$ and $10 \%$.

Morphology and BW QTL on LG4 were detected in the PHS as well as in the MHS analysis. The most likely location of the QTL was different in the two analyses: $55 \mathrm{cM}$ in the HS analysis compared with $0 \mathrm{cM}$ in the MHS analysis. However, the confidence intervals for QTL position were very large and overlapped, indicating that this might represent the same QTL. We can observe an interesting phenomenon between BW and morphological trait QTL; there were three BW-morphology QTL, one for PHS on LG4 and two for MHS on LG4 and LG6. For each pair of QTL, we noticed that both BW and morphology QTL were located at the same position in the linkage group. Body weight and all morphometric traits in this study were highly correlated, which might explain the observed link between both QTL (Volckaert et al. submitted).

\section{Discussion}

\section{Linkage map and information content}

The genetic map that we built resembles closely the previous genetic maps published by Chistiakov et al. (2005, 2008), with the difference that 23 new markers were added to our map. Although it is specific to our QTL population, the similarities between both of them show the consistency and relevance of our results. The map is of medium density with an average gap of about $8 \mathrm{cM}$, which is sufficient for QTL mapping according to Chistiakov et al. (2005).

The low IC detected in some regions on the linkage groups may impact the results, in particular for the QTL detected on LG4, which are numerous. Results in some regions become less reliable as the extremity of the chromosome is not very informative.

\section{Identification of QTL}

We found a total of 15 different QTL using the three analyses summarized in Table 3. The results found with VCA are similar to those found with MHS regression analysis. BW and morphology QTL were discovered at the same linkage groups and at similar positions; those traits were found to be highly positively correlated. Morphology QTL on LG4 explain 38\% of phenotypic variation, but some of the effects might be attributable to the actual BW QTL on the same linkage group. PHS detected more QTL, especially for the morphological trait, than either MHS or VCA. PHS and MHS differ in the number of QTL detected and in their position on the genome. More QTL were discovered for PHS. There are only two female parents included in the analysis, and this might have an impact on QTL discovery in MHS analysis. Potential QTL for MHS might not have been detected, because the only two females in the parental population did not segregate for this trait. The 95\% confidence intervals are very large for nearly all QTL, covering the entire length of chromosomes. Fine mapping, which is the next logical step in this process, must use a more dense genetic map to be able to pinpoint interesting genes, but our results are precise enough for a first QTL analysis. For fine-mapping, the use of more markers may help to reduce the confidence intervals and narrow the interesting regions that we found. At the moment, only microsatellites are available, but development of AFLPs (Chistiakov et al. 2008) and soon SNPs may accelerate the process of finemapping. Although the increase in the number of markers influences the precision of the results, population structure has an impact on the power to detect QTL. In this study, we have a mixed population of half-sib and full-sib. To have the best results, we selected the largest families. However, because of the unequal contribution of parents, the largest families will represent only very few parents.

The number of QTL found with the VCA is lower than with half-sib regression. This might be attributed to the 
Table 4 Body weight, morphological and cortisol QTL for paternal half-sib (PHS), maternal half-sib (MHS) and variance component analysis (VCA) of European sea bass.

\begin{tabular}{|c|c|c|c|c|c|c|c|c|c|c|c|}
\hline Trait & Method & $\begin{array}{l}\text { Position } \\
\text { in } \mathrm{cM}\end{array}$ & $\begin{array}{l}\text { No. segregating } \\
\text { parents }\end{array}$ & $\begin{array}{l}\text { Linkage } \\
\text { group }\end{array}$ & $\mathrm{F}$ & $\begin{array}{l}5 \% \mathrm{CW} \\
(\mathrm{HS})\end{array}$ & $\begin{array}{l}5 \% \text { GW } \\
(\mathrm{HS})\end{array}$ & LR & $\begin{array}{l}1 \% \mathrm{CW} \\
\text { (VCA) }\end{array}$ & $\begin{array}{l}\text { QTL } \\
\text { effect }(\%)^{1}\end{array}$ & $\begin{array}{l}\text { Confidence } \\
\text { Interval (cM) }\end{array}$ \\
\hline BW & PHS & 54 & 3 & 4 & 5.65 & 3.10 & 4.48 & - & - & 16 & $1-88$ \\
\hline BW & MHS & 0 & 2 & 4 & 7.09 & 3.76 & 7.02 & - & - & 8 & $0-76$ \\
\hline BW & VCA & 3 & - & 4 & - & - & - & 5.78 & 2.23 & - & $N A^{2}$ \\
\hline BW & MHS & 12 & 2 & 6 & 8.59 & 3.82 & 7.02 & - & - & 10 & $5-22$ \\
\hline MORPH & PHS & 55 & 3 & 4 & 4.76 & 2.90 & 4.18 & - & - & 13 & $1-105$ \\
\hline MORPH & MHS & 2 & 2 & 4 & 10.31 & 4.37 & 6.98 & - & - & 14 & $0-4$ \\
\hline MORPH & VCA & 2 & - & 4 & - & - & - & 15.46 & 13.03 & 38 & $N A^{2}$ \\
\hline MORPH & VCA & 19 & - & 6 & - & - & - & 22.79 & 12.07 & 9.4 & $N A^{2}$ \\
\hline MORPH & PHS & 5 & 2 & $1 \mathrm{~B}$ & 4.36 & 2.41 & 4.18 & - & - & 14 & $1-7$ \\
\hline MORPH & PHS & 30 & 3 & 7 & 5.71 & 2.60 & 4.18 & - & - & 16 & $0-34$ \\
\hline MORPH & PHS & 0 & 1 & 15 & 4.21 & 2.56 & 4.18 & - & - & 12 & $0-11$ \\
\hline MORPH & PHS & 0 & 2 & 24 & 4.93 & 2.50 & 4.18 & - & - & 13 & $0-6$ \\
\hline CORT & PHS & 3 & 2 & 3 & 2.69 & 2.70 & 4.59 & - & - & 8 & $0-13$ \\
\hline CORT & PHS & 1 & 2 & 14 & 3.01 & 2.74 & 4.59 & - & - & 9 & $0-59$ \\
\hline CORT & MHS & 3 & 1 & 23 & 6.59 & 3.25 & 7.61 & - & - & 10 & $0-9$ \\
\hline
\end{tabular}

BW, body weight; CORT, cortisol level; MORPH, combination of all morphometric traits (HD, HD, BD, BL, AL, PRAL, POAL and SL); PHS, paternal half-sib analysis; MHS, maternal half-sib analysis; VCA, variance component analysis; $c M$, centiMorgan.

${ }^{1}$ QTL effect: proportion of the phenotypic variation explained by the QTL.

${ }^{2}$ Not applicable.

approximate $1 \%$ chromosome-wide thresholds calculated with Piepho's method (Piepho 2001). Those thresholds are adjusted for the marker density of the genetic map.
Chatziplis et al. (2007) mention a QTL associated with morphometric traits located at the beginning of LG1; we also detected a morphology QTL on LG1. The two mapping
LG1b
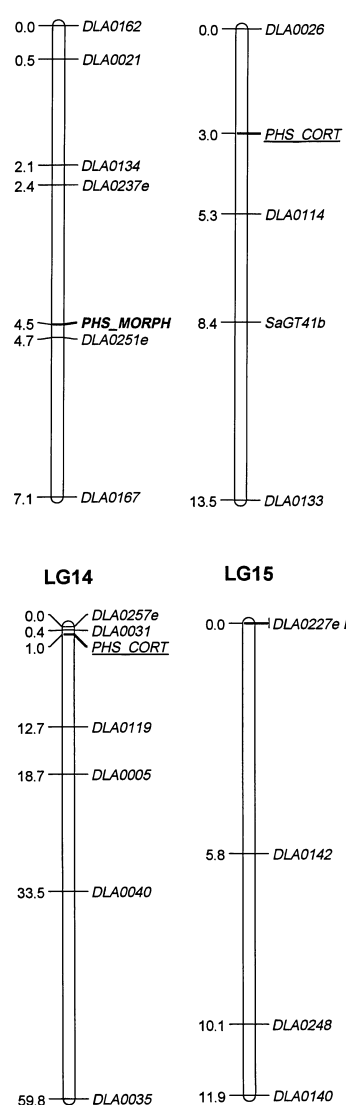

LG3

LG4

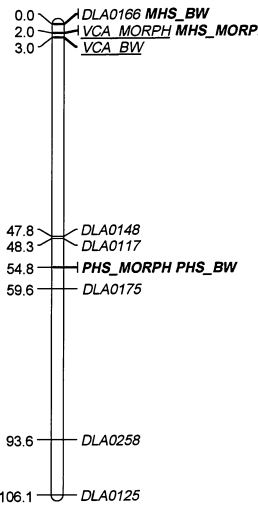

LG6

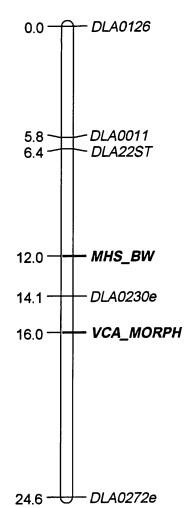

LG24

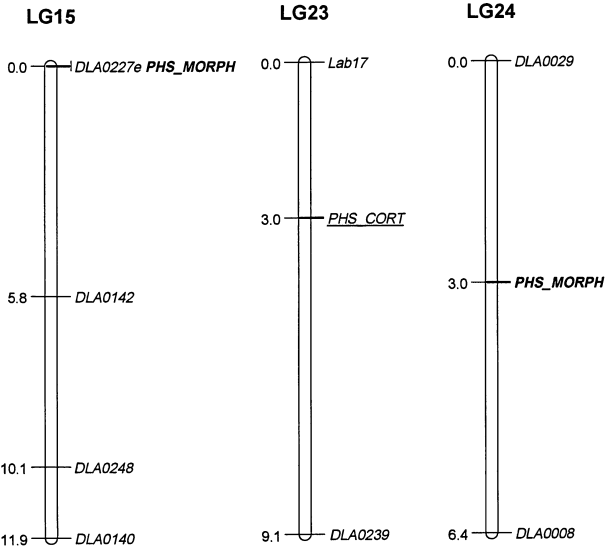

LG7

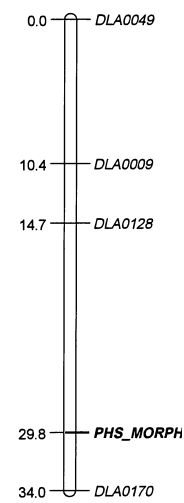

Figure 3 QTL in bold are 5\% genome-wide, whereas those underlined are $5 \%$ chromosome-wide. MHS stands for maternal half-sib regression, PHS for paternal half-sib regression and VCA for variance component analysis. The traits are MORPH for morphology and BW for body weight.

.


populations differ, although they belonged to the same company. We split this linkage group into LG1a and LG1b because of a large region in the middle of LG1 devoid of markers. Our QTL is located at the end of the linkage group, in the LG1b section. The high confidence intervals suggest that the location of the QTL could be anywhere on the chromosome, and therefore this result potentially confirms the results of Chatziplis et al. (2007). These authors also mention a possible BW QTL on LG1, but we could not support this finding. No other QTL were reported in European sea bass, suggesting that we found novel significant and suggestive QTL for this species.

We found three suggestive QTL for stress response. Although they are not highly significant, it is an important discovery, because QTL for stress response have not been reported before. A candidate gene for this trait could be the glucocorticoid receptor, which has been sequenced and whose position remains unknown (Terova et al. 2005). Several genes related to carbohydrate metabolism, genes involved in $\mathrm{Na}^{+} / \mathrm{K}^{+}$transport, polyamine biosynthesis and iron homeostasis (Sarropoulou et al. 2005) could be also taken as candidate genes, as they are involved in the mechanism of stress response. Our identified regions could be of high interest for candidate gene approach studies.

\section{Methodological comparison}

We compared half-sib regression and VCA, and each method had its advantages and drawbacks. We performed separately a PHS regression analysis and MHS regression analysis. Therefore, in the regression analysis, the full-sib relationships are not taken into account. We additionally performed a VCA that deals with all family relations in our experimental population. Half-sib regression does not require large computing resources and time, and therefore it allows a permutation test to be performed and confidence intervals to be calculated using bootstrapping.

Variance component analysis has been implemented to simultaneously account for all relations in our data. The QTL is modelled as a random effect in the model; therefore, the QTL may have an infinite number of alleles. However, VCA is computationally demanding and time consuming, especially for the calculation of IBD and thresholds for 5\% chromosome-wide significance when computed according to an approximation given by Piepho (2001).

\section{Power of the experimental design}

This study has implemented the two-step procedure developed by Massault et al. (2008). When applying simulated parameters from our specific population (rounded to 600 individuals), we found that, for an $80 \%$ power, we were theoretically able to detect QTL explaining at least $14.5 \%$ of phenotypic variation using half-sib regression analysis and at least $6.5 \%$ of phenotypic variation using VCA. Mor- phology QTL on LG4 explain a large proportion of phenotypic variation (38\%) and will have been detected with a less powerful experimental design. In the case of PHS regression, this QTL could be associated with marker DLA0175, whereas for MHS and VCA, it will be more linked to a marker at the beginning of the linkage group DLAO166. In contrast, morphology QTL on LG6 might not have been detected, as the QTL effect was $9.4 \%$ of the phenotypic variation (against 14.5\% expected theoretically). Our design was powerful enough to detect a QTL of medium size, which is proof that the design is perfectly adapted for a natural mating population. The proportion of phenotypic variation explained by QTL detected with half-sib regression was of medium size effect $(8-16 \%)$. This shows that our method is able to detect not only QTL with large effects, but also medium effects for sea bass, and could be generalized to mass-spawning species.

The fact that there were only two female parents involved in the experiment may also have an impact on the success of detecting QTL. The heterozygosity for each allele is therefore none, half or one. As explained earlier, existing QTL might be ignored because none of the females are segregating for them. The power therefore might be lower, as in theory we assumed a heterozygosity of 0.5 , which in reality is impossible to predict.

The theory was based on a sparse genetic map, with marker spacing of $20 \mathrm{cM}$. Our genetic map had average marker intervals close to $8 \mathrm{cM}$. A denser map could have improved the power, and therefore our experiment might not have detected some QTL.

The power of the experiment depended on the heritability of the trait. We found three suggestive CORT QTL for stress response. The heritability of this trait shown in Table 1 is 0.08. Massault et al. (2008) have set a high heritability of 0.5 for the two-step experimental design. Trait heritability is known to play a role in QTL discovery (Kolbehdari et al. 2005), and in the case of low heritability such as cortisol, there is less chance of detecting important QTL.

Our study shows that QTL mapping is possible for natural mating mass-spawning species, using a specific experimental design and tools such as parentage assignment. The results of this experiment are preliminary, but they are promising. QTL were detected and could be located at specific chromosomes, so that complementary fine-mapping can be undertaken.

\section{Acknowledgements}

This study has been funded by the European Commission (project AQUAFIRST, contract number FP6-STREP2004-513692). DJK and CSH acknowledge financial support from the BBSRC. E. Couto, S. Darivianaku, J. Fuentes, P. Guerreiro, M. Kampakli, G. Kotoulas and D. Power helped with phenotyping on-site. 


\section{References}

Chatziplis D., Bartagias C., Tsigenopoulos C.S., Magoulas A., Kollias S. Kotoulas G., Volckaert F.A.M. \& Haley C.S. (2007) Mapping quantitative trait loci in European sea bass (Dicentrarchus labrax): the BASSMAP pilot study. Aquaculture 272, S172-82.

Chistiakov D.A., Hellemans B., Haley C.S., Law A.S., Tsigenopoulos C.S., Kotoulas G., Bertotto D., Libertini A. \& Volckaert F.A.M. (2005) A microsatellite linkage map of the European sea bass Dicentrarchus labrax L. Genetics 170, 1821-6.

Chistiakov D.A., Tsigenopoulos C.S., Lagnel J., Yuanmei G., Hellemans B., Haley C.S., Volckaert F.A.M. \& Kotoulas G. (2008) A combined AFLP and microsatellite linkage map and pilot comparative genomic analysis of European sea bass Dicentrarchus labrax L. Animal Genetics 39, 623-34.

Dupont-Nivet M., Vandeputte M., Haffray P. \& Chevassus B. (2006) Effect of different mating designs on inbreeding, genetic variance and response to selection when applying individual selection in fish breeding programs. Aquaculture 252, 161-70.

Dupont-Nivet M., Vandeputte M., Vergnet A., Merdy O., Haffray P., Chavanne H. \& Chatain B. (2008) Heritabilities and GXE interactions for growth in European sea bass (Dicentrarchus labrax L.) using a marker-based pedigree. Aquaculture 275, 81-7.

Engelsma M.Y., Huising M.O., van Muiswinkel W.B., Flik G., Kwang J., Savelkoul H.F.J. \& Verburg-van Kemenade B.M.L. (2002) Neuroendocrine-immune interactions in fish: a role for interleukin-1. Veterinary Immunology and Immunopathology 87, 467-79.

García de León F.J., Dallas D.J., Chatain B., Canonne M., Versini J.J. \& Bonhomme F. (1995) Development and use of microsatellite markers in seabass, Dicentrarchus labrax (Linnaeus, 1758) (Perciformes: Serranidae). Molecular Marine Biology and Biotechnology 4, 62-8.

George A., Visscher P. \& Haley C.S. (2000) Mapping quantitative trait loci for complex pedigree: a two-step variance component approach. Genetics 156, 2081-92.

Heath S.C. (1997) Markov chain Monte Carlo methods for radiation hybrid mapping. Journal of Computational Biology 4, 505-15.

Herlin M., Delghandi M., Wesmajervi M., Taggart J.B., McAndrew B.J. \& Penman D.J. (2008) Analysis of the parental contribution to a group of fry from a single day of spawning from a commercial Atlantic cod (Gadus morhua) breeding tank. Aquaculture $274,218-24$

Hernandez J. \& Knott S. (2009) Haplotyping via minimum recombinant paradigm. BMC Proceedings 3(Suppl. 1), S7.

Jones M.W. \& Hutchings J.A. (2002) Individual variation in Atlantic salmon fertilization success: implications for effective population size. Ecological applications 12, 184-93.

Knott S., Elsen J. \& Haley C.S. (1996) Methods for multiple-marker mapping of quantitative trait loci in half-sib populations. Theoretical and Applied Genetics 93, 71-80.

Kolbehdari D., Jansen G.B., Schaeffer L.R. \& Allen B.O. (2005) Power of QTL detection by either fixed or random models in halfsib designs. Genetics Selection Evolution 37, 601-14.
Massault C., Bovenhuis H., Haley C.S. \& de Koning D.J. (2008) QTL mapping designs for aquaculture. Aquaculture 285, 23-9.

Moretti A. (1999) Manual on Hatchery Production of Seabass and Gilthead Seabream. Food and Agricultural Organisation, Rome.

Piepho H.P. (2001) A quick method for computing approximate thresholds for quantitative trait loci detection. Genetics 157, 42532 .

Saillant E., Chatain B., Forstier A., Przybyla C. \& Fauvel C. (2001) Parental influence on early development in the European sea bass. Journal of Fish Biology 58, 1585-600.

Saillant E., Dupont-Nivet M., Haffray P. \& Chatain B. (2006) Estimates of heritability and genotype-environment interactions for body weight in sea bass (Dicentrarchus labrax L.) raised under communal rearing conditions. Aquaculture 254, 139-47.

Saillant E., Dupont-Nivet M., Sabourault M., Haffray P., Laureau S., Vidal M.O. \& Chatain B. (2009) Genetic variation for carcass quality traits in cultured sea bass (Dicentrarchus labrax). Aquatic Living Resources 22, 105-12.

Sarropoulou E., Kotoulas G., Power D.M. \& Geisler R. (2005) Gene expression profiling of gilthead sea bream during early development and detection of stress-related genes by application of cDNA microarray technology. Physiological Genomics 23, 18291.

Scott A., Sheldrick E. \& Flint A. (1982) Measurement of 17[alpha], 20 [beta]-dihydroxy-4-pregen-3-one in plasma in trout (Salmo gairdneri Richardson): seasonal changes and response to salmon pituitary extract. General and Comparative Endocrinology 46, 44451

Souche E. (2009) Genomic Variation in European Sea Bass Dicentrarchus labrax: From SNP Discovery Within ESTs to Genome Scan. PhD Thesis, Katholieke Universiteit Leuven, Leuven, Belgium.

Terova G., Gornati R., Rimoldi S., Bernardini G. \& Saroglia M. (2005) Quantification of a glucocorticoid receptor in sea bass (Dicentrarchus labrax, L.) reared at high stocking density. Gene $357,144-51$.

Vandeputte M. \& Launey S. (2004) The genetic management of fish domestication. Productions Animales 17, 237-42.

Vandeputte M., Dupont-Nivet M., Chatain B. \& Chevassus B. (2001) Setting up a strain-testing design for the seabass, Dicentrarchus labrax: a simulation study. Aquaculture 202, 329-42.

Vandeputte M., Dupont-Nivet M., Chavanne H. \& Chatain B.A. (2007) Polygenic hypothesis for sex determination in the European sea bass Dicentrarchus labrax. Genetics 176, 1049-57.

Volckaert F.A.M., Batargias C., Canario A., Chatziplis D., Chistiakov D., Haley C., Libertini A. \& Tsigenopoulos C. (2008) European sea bass. In: Genome Mapping and Genomics in Animals (Ed. by T.D. Kocher \& C. Kole), Volume 2: 117-33. Springer-Heidelberg, Berlin.

Volckaert F.A.M., Hellemans B., Batargias C., Louro B., Massault C., Van Houdt J.K.J., Haley C., de Koning D.J. \& Canario A.V.M. (submitted) Family assignment and heritability of body weight, morphometric traits and stress response in aquacultured European sea bass Dicentrarchus labrax. 